\title{
EduGram: Education Development Based on Hologram Technology
}

\author{
https://doi.org/10.3991/ijoe.v17i14.27371
}

\author{
Asma S. Ahmad ${ }^{1}$, Alaa T. Alomaier ${ }^{1}$, Doaa M. Elmahal ${ }^{1}$, Reem F. Abdlfatah ${ }^{1(凶),}$ \\ Dina M. Ibrahim ${ }^{1,2}$ \\ 'Department of Information Technology, College of Computer, \\ Qassim University, Buraydah, Saudi Arabia \\ ${ }^{2}$ Computers and Control Engineering Department, Faculty of Engineering, \\ Tanta University, Tanta, Egypt \\ reemfahmi9@gmail.com
}

\begin{abstract}
Education is one of the most important areas of life that affect the development and progress of societies, and the usage of images and visual representation methods is of real value in the educating process. Over time, different simple methods were used to display the information visually, which mostly are considered weak methods that may not perform its full purpose and the information may be transferred slowly and in an incomplete manner. Technology has contributed from the beginning of its emergence in the development of education and improve its output, and one of the most prominent contributions made by technology is the developments in the field of displaying information visually using different technologies, as the three-dimensional displaying technologies that are considered as an advanced solution which provides people with a more comprehensive view and facilitates the task of transferring information to learners and so improving the educating process. Many technologies are used to create and display the 3D visual content, and two of the most important 3D display technologies are Augmented Reality technology and Hologram technology, they both insert a three-dimensional image to the real world, but there are many differences between the two technologies in many aspects. In the first part of this research, and after defining and comparing the two technologies and the effect of each one on education, Hologram technology showed features that enabled it to be a suitable option to be used in education for displaying 3D educational content. This research then introduces the method for implementing the usage of Hologram technology in education as a 3D educational content displaying tool, introducing an implementation model by first transferring a sample of a 2D educational image to Holograms and using the Hologram fan projector to display it to the students. The results of a simple questionnaire on a number of people showed the effectiveness of using Holograms instead of the traditional 2D content found in school curricula, and a good level of people's acceptance to use this technology.
\end{abstract}

Keywords — hologram, education, augmented reality, three-dimensional image, 3D hologram fan 


\section{Introduction}

Since prehistory and ancient times, education with its different types and methods was one of humanity's most important functions, and education is still in constant development and investment in developing it is considered one of the most successful businesses these days; due to its importance to societies development, and the improvement of people's lives in general, and it is defined as the process of transferring information and concepts to learners in order to increase their wealth of knowledge and guide them, using a variety of information transfer methods [1] [2].

The use of images and visual representation methods for the promotion of scientific content is of real value, as the Chinese philosopher Xunzi said: "Not having heard something is not as good as having heard it; having heard it is not as good as having seen it." [3]. The images help us learn, and it attracts attention, explains, inspires tough concepts, and contributes to the stability of information [4]. A lot of visual communication methods and technologies were used to present the information, onset from primary methods such as drawing, graphic illustrations, two-dimensional (2D) images, and videos to the usage of three-dimensional models (3D) that are more realistic [5].

Technology plays a significant role in education development, it has changed the learning environment and gave teachers other sophisticated ways to teach students, the integration of technology in education has positively affected both the learners and instructors, it enhances controlling, information transfer, and the acquisition of this information by learners [6]. Information imaging was the most processes that needed for the usage of technology, different techniques were used and still in use for the presentation of information visually, 2D and 3D images, and different visual multimedia was generated using technology' various tools.

As technology continues to progress, we are given the opportunity to explore new and more useful types of techniques that offer solutions and enhancements in people's lives, one innovation that is growing at a rapid pace is Hologram technology. Hologram technology is the technology that displays 3D images created using light diffraction, and these images are able to be seen by the naked eye, this feature makes Hologram technology a very useful and adequate technology to be used in many different fields to develop viewing methods, specifically in education as an educational tool [7].

This study arises from a previous one, carried out by the authors, related to the study about Hologram technology and Augmented Reality technology [8]. Here, we are aiming to develop education through the insertion of Hologram technology as a good tool for information illustration, using Hologram technology, the instructor can transfer the information smoothly and easily to learners, also, the learners are able to gain the information more quickly and with an attractive method, which will eventually result in a successful educational experiment [9].

\section{Problem specification and motivation}

Education is an important field and every problem that affects it will return with negative results, not for the learners only but the effect can reach the whole society, so it was important to focus on it and develop its methods. The traditional way of educating 
includes a number of different methods of information transferring usually under the guidance and depending on educators and their verbal description to explain the information and with the use of two-dimensional visual content, as shown in Figure 1, these same methods can affect students understanding ' time and their ability to recognize the information; that is because the insufficient explanation of information and the bad, unattractive educational content, as well as the problem of instructor inability to transfer the knowledge clearly, effects the educating process negatively and this may result in the fall of the entire educating process, which is not the thing desired to occur [1] [10].

Humans are visual creatures and a large percentage of the human brain dedicated itself to visual processing they seek the easiest and most fluent way of acquiring and learning information, so it was necessary to concentrate on the process of clarifying the information visually and develop the visual contents in their education [4] [11], also, the humans' perception is three-dimensional, as when they look at an object, the retina in each eye forms a two-dimensional image of our surroundings and the brain processes these two images into a 3D visual experience [12]. As Figure 1 illustrates, the three-dimensional visual content is considered to be a proper solution that solves the problems in the traditional visual methods used to display the information, as it conforms with the way people see things, it gives the learners a larger view and more information about the image as if it is a real existing object; which contributes to the development of the process of conveying information properly to learners and providing visual content that depicts reality in a more accurate and clear way [13] [14].

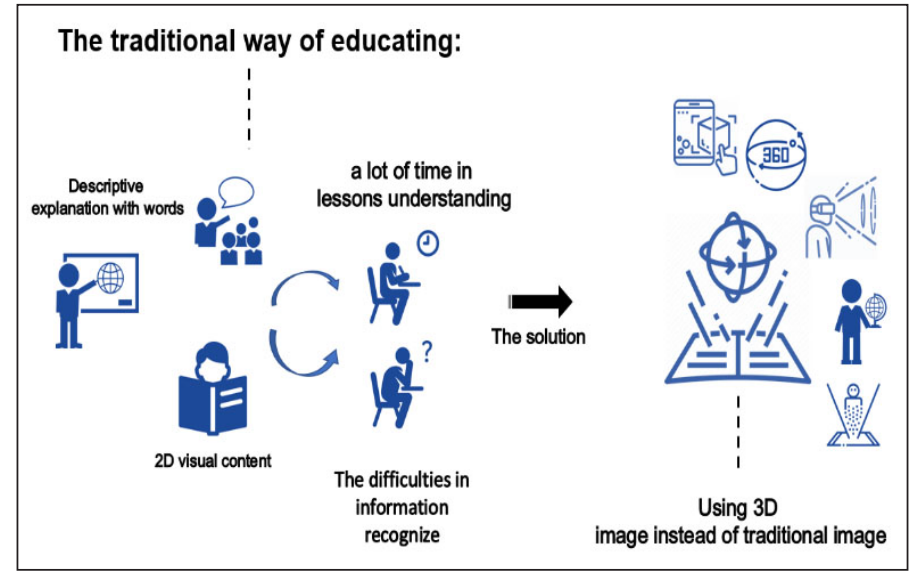

Fig. 1. Graphical illustration for the problems in the traditional education methods and the suggested solution

Hologram technology — which is one of the technologies that displays 3D imageshas many features as the high ability to display the information in a more real, clear, and attractive manner that qualify it to be a good alternative to the traditional method of information illustration [7]. In addition to this, from the research conducted, it was clear that the presence and usage of Hologram technology are largely limited to advertising and entertainment despite its high features which may benefit most fields in the world 
as the process information transferring visually in education, so, the insertion of it in order to develop the education may be considered a good and useful addition to both fields [15].

\section{Literature review}

As it is obvious, that education is considered as an important field that always needs to be enhanced and developed, also, based on the nature of humans; the educational visual content is the preferred way to transfer information to the learners easily and quickly. The effectiveness of the usage of three-dimensional visual content and its superiority over the traditional methods used to provide information visually had been proven, and this is because it facilitates the viewing of the objects and getting more details from the image; which is consistent with human $3 \mathrm{D}$ visual perception. The technology presented a number of techniques that provide users with three-dimensions images, and based on their features; the most two suitable technologies to be used in education are Hologram technology and augmented reality technology, as they are shown in Figure 2, they are technologies that add and display $3 \mathrm{D}$ images in the reality surrounding the learners.

\section{Options to implement the usage of the $3 \mathrm{D}$ models}

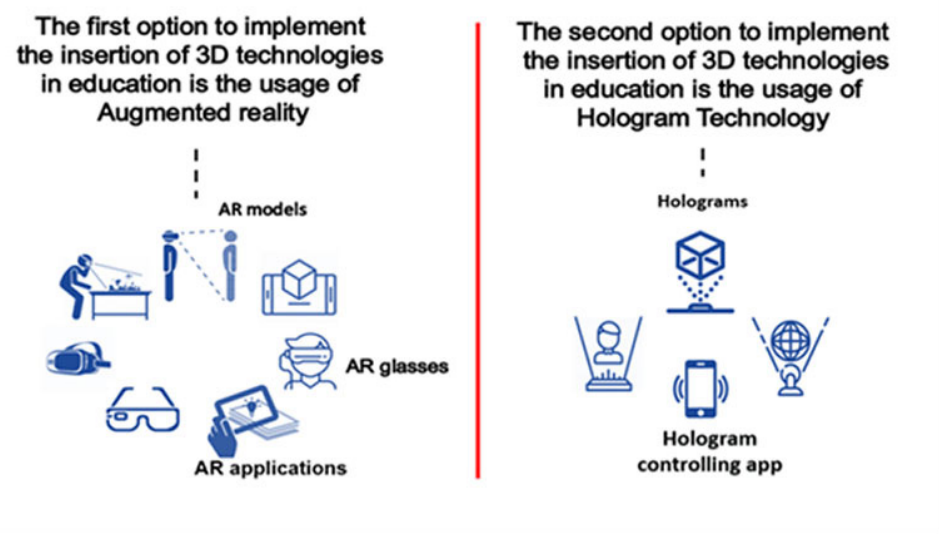

Fig. 2. The options to implement the insertion of the $3 \mathrm{D}$ visual content in education

Augmented reality and Hologram technology appear as if they were the same technology, and people usually confuse them, but they differ from each other and each one is a different technology [8]. The suggestion of this research declares that Hologram technology is considered to be the proper tool to display the $3 \mathrm{D}$ visual information, so, in order to prove its effectiveness and then determine the method to implement the usage of it in education properly, it was necessary to define it, and Augmented reality the technology that resemblance it, then differentiate between them and compare them 
when they are used in education, after that, it will be possible to define the method by which the Hologram technology can be used as a displaying tool in education.

\subsection{Background of hologram and augmented reality technology}

A Hologram (that is pronounced as HOL-O-Gram), is a particular type of three-dimensional image that can be seen with just eyes and it exists really in space what makes it visible to everyone without the need of 3D glasses [16] [17] [18]. Hologram gets its names from Greek words meaning "whole message" which is coined by Dennis Gabor [19].

Figure 3 shows the most important events that took place for Hologram technology development [8].

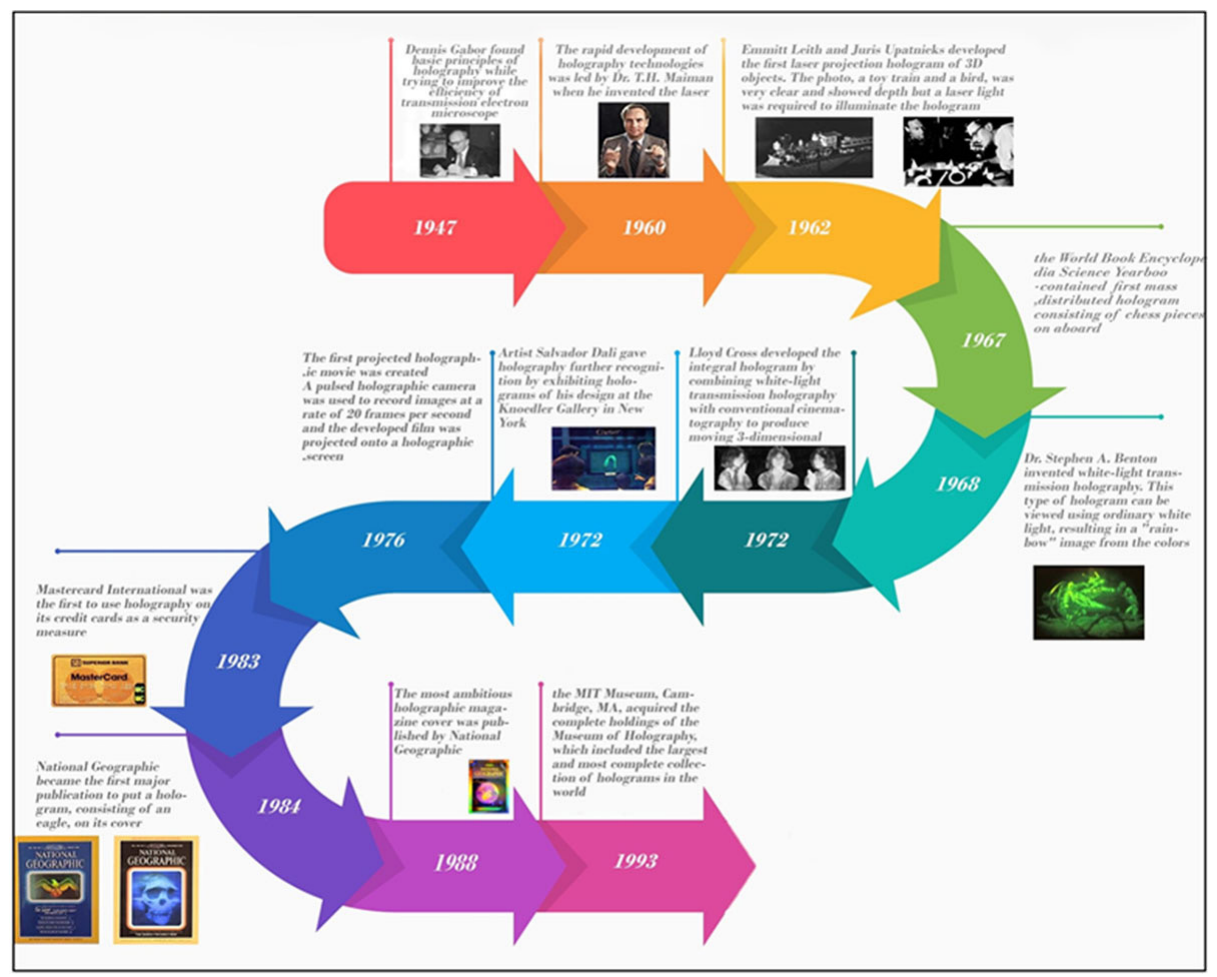

Fig. 3. Hologram technology timeline

Augmented reality is a technology that can display three-dimensional objects but it can't be seen by naked eyes. It offers an enhanced version of the real physical world through the use of unphysical 3D objects [20] [21]. 


\subsection{Comparison between hologram technology and augmented reality technology when used as a tool for education}

As it is stated, Hologram technology and augmented reality technology produce almost the same result; as they add a three-dimensional image to the surrounding reality, but in fact, each of them is a separate technology that differs in the way it creates the image, the way it is displayed, its types, and the hardware and software needed. So, in order to compare between them and then being able to decide which one is suitable to be used in education:

- Hologram Technology/Augmented Reality Images:

Hologram technology and Augmented Reality technology both give the users a view of a $3 \mathrm{D}$ image that is floating in reality, but this created image largely differs in its properties and the method of creation. The effect of the presence is the biggest difference between the two technologies, as the 3D image in Hologram technology is displayed through a programmed projector that projects the $3 \mathrm{D}$ image using the reflections of light or the laser beams in a physical medium, so the image really exists and there is no need to wear special glasses or seeing the 3D image inside any screen, while in Augmented Reality the 3D image is totally virtual programmed image that is augmented in the real world with the help of a camera in the smart device and then displayed in its screen [8] [22].

Figure 4 [23], shows the 3D image (a Hologram) displayed in Hologram technology, and Figure 5 [24] shows the image displayed in Augmented Reality.

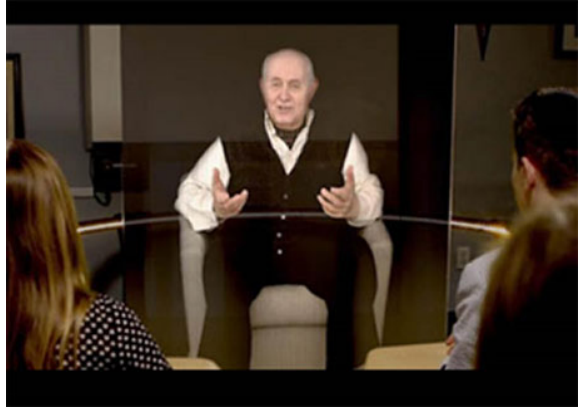

Fig. 4. The image in hologram technology

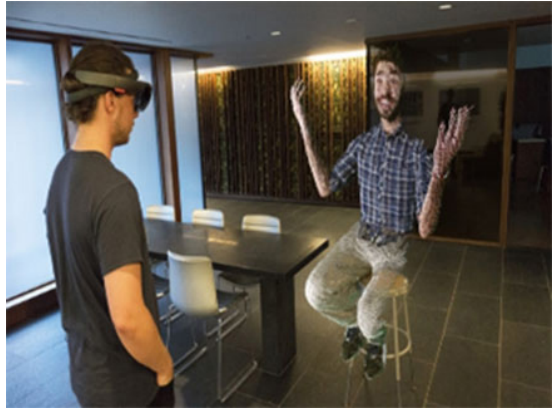

Fig. 5. The image in augmented reality

On the other hand, the resolution of the image in Augmented reality may sometimes exceed the resolution of the Holograms displayed with a Hologram technology projector; owing to the effect of the air and environment in which the Holograms are displayed, therefore, the surrounding environmental conditions must be taken into consideration when using the Hologram technology to ensure the quality of the displayed image and the satisfaction of viewers. But, the surrounding conditions in the classroom are often fixed and initialized in the beginning in order to avoid any error that may affect the educational content displayed, so this defect may not affect significantly on education when using Hologram technology in schools. 
- Hologram Technology/Augmented Reality Types:

Many types fall under both Augmented Reality technology and Hologram technology and the classifications that underlie each type also differ. Under this comparison aspect, Hologram technology excels on Augmented Reality technology; because of its different multiple types and so high flexibility, and so it could be implemented and used with the method that suits capabilities and meets the requirements [8] [25].

- Hologram Technology/Augmented Reality Implementation and Use Requirements:

The specification of requirements is very important to determine what the users need from this system and what must be provided and to ensure that the desired goals will be achieved, and the requirements largely differ according to users' differences, environments, and different technologies used. The requirements that must be specified when using Hologram technology or Augmented Reality technology are commonly classified into user requirements, software requirements, and hardware requirements:

User Requirements: For the systems of 3D displaying technologies, the users mostly require the same visual properties, as humans have a 3D perception when they look at an object so it is important for them to get a semi-real image when communicating visually using these technologies [8].

In education, the users of the Hologram technology and AR systems are the instructors who control the learning process and the learners to whom the information is transferred to visually, so given the conditions in which education takes place, the users in education require additional needs that must be taken into account.

From the graph, it can be concluded that the use of Hologram technology is the most appropriate option that satisfies most of the users' needs in an acceptable limit, especially in the case that the number of students is somewhat large, as it will be the most cost-effective, and easiest in controlling the presentation of the content for the teacher.

Software Requirements: The basic software requirements needed to operate both Hologram technology and Augmented Reality technology are somewhat similar, which are: An operating system, specialized programming language, and three-dimension images editing and designing software. Hologram technology, on the other hand, is very flexible in the type of software needed [8].

Hardware Requirements: Hologram technology and Augmented Reality technology both mainly need a hardware device to view the objects. And there is hardware that is needed by both Augmented Reality and Hologram technology which is the smart devices, and sometimes cameras, in contrast, the two technologies differ in the needed hardware that displays the 3D object, as each one requires its specialized hardware that projects the image in the user's environment [8]. Augmented Reality requires either AR glasses that enable the user to see the virtual 3D image when wearing it or a smart device such as smartphones that view the virtual 3D image on its screen, where Hologram technology needs only a special projector that creates Holograms and displays it in the surround [8].

For the students to be able to see the 3D images in Augmented Reality, every single user must have either a smart device or AR glasses [26] and this result in two problems: 
1. It very costly to offers an AR viewer for all students in the school.

2. The controlling of the educating process will be difficult for the instructor and synchronize his speech with what is presented in the students 'devices, as well as the difficulty of following up on each student separately.

In the case of Hologram technology been used, the hardware needed to display the $3 \mathrm{D}$ image for all student is just a single Hologram projector [27], which is considered a cost-effective option and the instructor will able to control the education process easily and be able to make sure that all students receive the knowledge synchronously.

Briefly, Figure 6 displays the relationship between the student numbers and the level of users (students and teacher) satisfaction when using Augmented reality or Hologram technology, as it is obvious when the number of students is reasonably little, both Augmented reality and Hologram technology will be suitable to be used, but as the number of students increases the effectiveness of Hologram technology will increase over Augmented reality.

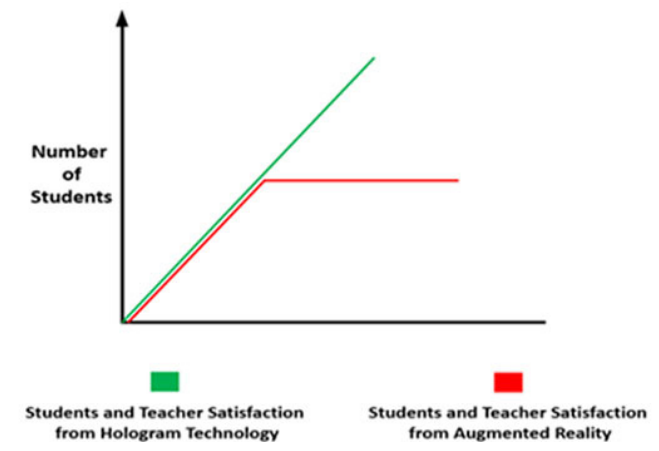

Fig. 6. Relationship between student numbers and users satisfaction

\subsection{The usage of hologram technology in education "EduGram"}

From the previous comparison between Hologram technology and Augmented reality, it is obvious that Hologram technology almost outperformed the Augmented reality technology in terms of its suitability to be a good educational tool. Based on the above comparison and as shown in Figure 6, when the number of learners is large or increased which is the common situation, there will be problems with the use of Augmented reality technology, which will negatively affect the course of the educational process. On the other hand, Hologram technology showed its distinction as it provides many features that meet the needs of most learners with the lowest requirements this enables it to be the proper choice that replaces Augmented reality in education, and some of the important features that Hologram technology offer are:

1) The flexibility to choose the right type to be used.

2) The ease of control and display of content.

3) Cost-effectiveness.

4) The satisfaction of a large number of users.

5) Display content to all learners at the same time, which results in the smooth operation of the instruction process. 
After it has been proven that Hologram technology is considered the appropriate tool for displaying the educational visual content, this which supports the proposal of this research and so the aim of the research (developing education using Hologram technology) could be adopted. As shown in Figure 7 the general proposal of this research is the "EduGram" system - taken from the words Education and HoloGram-the system to integrate the Hologram technology in education as a displaying tool that offers the learners a 3D educational visual content, and its usage is combined to the ordinary teaching methods that are conducted under control and guidance of an instructor to a number of learners, and which is hoped from it that it will produce a successful learning experience.

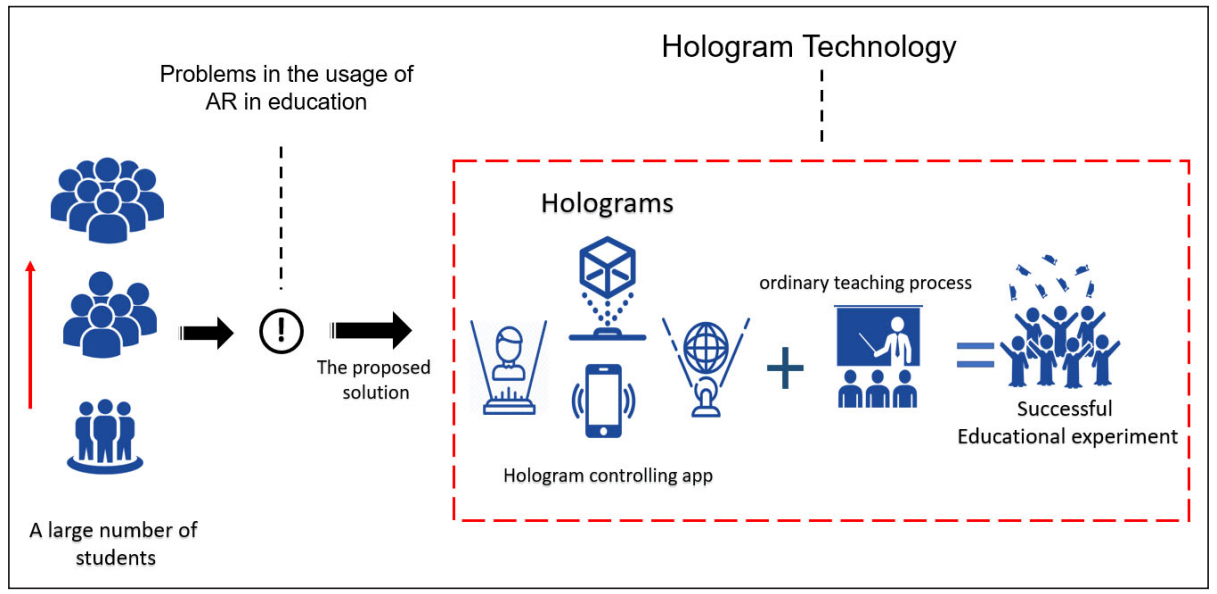

Fig. 7. Usage of hologram technology in education

\section{Methodology}

Below are the implementation details of the process of developing the education process using Hologram technology for EduGram system, starts by identifying the procedure to implement and insert the Hologram technology in education in general, then the implementation requirement and sampling are specified, after that, the chosen structure to implement EduGram system in this research will be identified:

\section{- EduGram Procedure:}

The procedure to insert the Hologram technology in education-which we referred to as EduGram system - in order to develop education as proposed in this research is through using a Hologram projector under the supervision of the instructor as an educational tool that displays the visual information that must be explained to learners in the form of a Holograms, , that is while carrying out the information transfer process, the instructor uses the Hologram projector to display the three-dimensional visual information existed in the lesson he is currently teaching or that which included in any knowledge currently transferring to learners. 
- EduGram Implementation Requirement and Sampling:

To implements any Hologram technology system there are three core requirements so that the Holograms (the 3D images) could be displayed which are:

1) The Hologram projector: the core element in the system, which is responsible for projecting the Holograms.

2) Controlling method: either hardware and software or just a software that is used to control the displaying of Holograms in the Hologram projector.

3 ) Visual content: the visual content to be projected by the Hologram projector.

\section{EduGram's Hologram Projector Device (Hologram fan):}

As stated previously, Hologram technology requires a projector device to project the Holograms in the users' circumference so it can be seen by the naked eye. and there are many different types of Hologram projectors that project really existing 3D images with different properties depending on the way the projector device works [28].

The Hologram projector type chosen to implement the EduGram system is the Hologram fan projector, which is an advanced new type of projector that creates the illusion of a Hologram that is floating in the air using an optical illusion technique called POV (Persistence of Vision), these Holograms are projected using a large number of tiny bright light-emitting diodes built-in the fan blades and which switch light colors in milliseconds. Hologram fan comes with either two or four blades, the LEDs on each blade are programmed so that every light is emitted at a specific time and in a specific order to display the complete Hologram, the fan also contains a core control unit that acts as a fan's CPU, which the blades are attached to, and which also contains power port and a port to insert the memory card that stores the 3D multimedia that will be displayed. The Hologram fan works with electricity power and once the fan is turned on, the blades start to spin quickly to the extent that it appears invisible, and the control unit lighting up the LEDs as programmed to create a floating Hologram that is updated every millisecond to give users a stable complete 3D images [28] [29].

Hologram fan projector is characterized by many of the advantages that qualified it to be a suitable option to be used in EduGram system to develop education, such as: easy installation, easy usage as no technical skills are needed to use it, easy flexible controlling, global availability, low price, variety of shapes and technological capabilities, the possibility of using several accessories such as the holder and covers in proportion to the requirements of use, and supporting of several technologies such as Bluetooth and Wi-Fi. Figure 8 shows the separated parts and the installed situation of the "Holo65 3D Hologram Fan Display upgraded fan model" from "GIWOX" company used in this research in order to implement the EduGram system, which is characterized by that it has good capabilities and a reasonable price qualified it to be a suitable option that commensurate with our capabilities. 


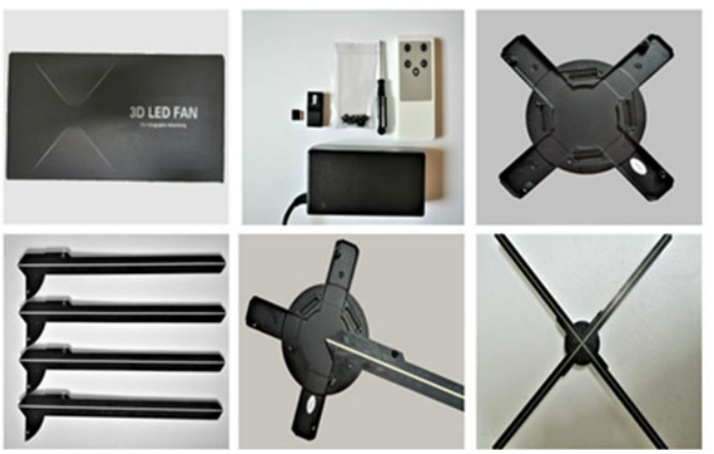

Fig. 8. EduGram hologram fan

The "Holo65 3D Hologram Fan Display" is one of many models that the company sells, it costs around 7200\$ in Amazon.com. The fan consists of four blades with 960 Led Beads. The fan size is 25.6 Inch which considered somewhat large, it is upgraded on October 16, 2019, so that now it offers more resolution 960P rather than 720P in the old models. Also, it offers High-definition 3D holographic visuals with sounds where Bluetooth 4.0 support was added. The fan also is supporting WIFI technology as it is controlled using smartphone devices, and the device has the ability to be integrated on a Hologram wall to produce larger Holograms [30].

After installing the basic parts of the device and in order to obtain the best way to use it, the device must be positioned in a fixed place so that it is safe to use and comfortable to view and easy to deal with. In order to implement the usage of Hologram technology in education properly, we decided to use the fan stand which we manually made, taking into consideration its hardness and durability, and how long it must be so it will be proper and comfortable for both the teacher and students while conducting the lesson, The left part in Figure 9 shows the device after we position it into the stand, and the right one shows the Hologram fan device after it operates which is displaying a headphone's Hologram.
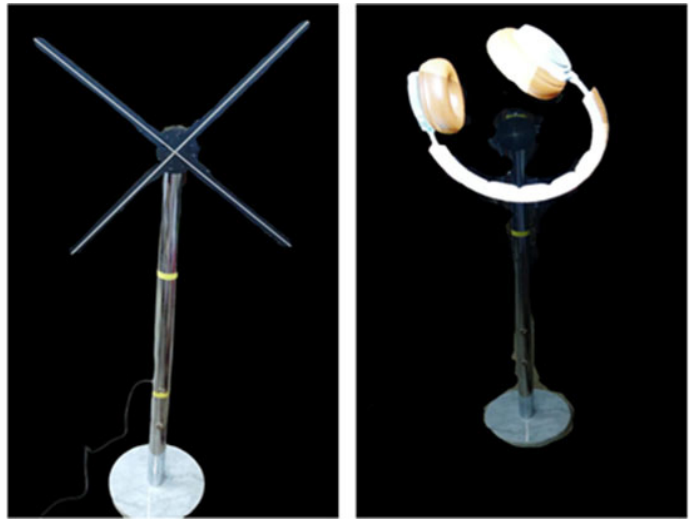

Fig. 9. EduGram hologram fan 


\section{EduGram Controlling Method:}

There are different methods to control the Hologram fan device displaying, it can be controlled either by a remote control which has only a few primary essential controls functions, or using specialized software programs installed in a device that has higher capabilities and advantages.

Different programs are used to control the fan device according to the different fan models manufactured in each company, and some models support the controlling through the cloud system which enables the user to remotely control the Hologram fan device through the internet and which considers a more developed method for device control. "Holo65 3D Hologram Fan Display" used to implement EduGram system, is capable to be controlled with the remote-control device offered with the fan device, or using its specialized smartphone application that is developed by "HeRui" applications developers. The name of the application is "3D LED FAN" and its last version is the 3.2 .5 edition.

\section{EduGram Three-Dimensional Content:}

We looked at the educational content in the school's textbooks, then we chose the science subject as it contains a lot of important visual information that was poorly explained, the science book contains most of the vital information from body parts, cell configurations, and other vital processes, which is considered one of the most important and complicated visual contents, therefore, it is an appropriate field to select content from it and converts it into 3D models. We selected the best three topics from the book and focused on it, initially, to facilitate the implementation and obtain more accurate results. Figure 10 displays the selected visual content as shown in the school textbook, (a) shows the figure of "Human Heart", (b) is the "Human Eye" figure, and (c) display the "Cell Division".

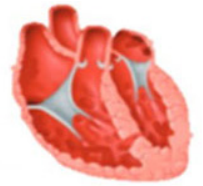

(a)

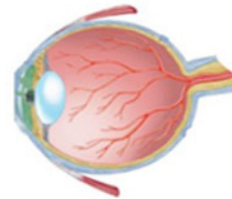

(b)

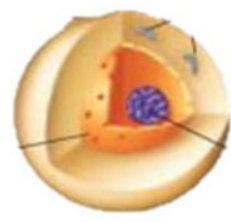

(c)

Fig. 10. Old used educational visual content

We designed the educational content that we chose as 3D models, and we took into account the clarity of colors and the different parts of the model and its realism. Figure 11 displays our 3D models that will replace the previous picture used on the school's books, on (a) the 3D model (a Hologram) of the human heart, (b) shows the 3D model (a Hologram) of the human eye, and in (c) the 3D model (a Hologram) of the split cell. 


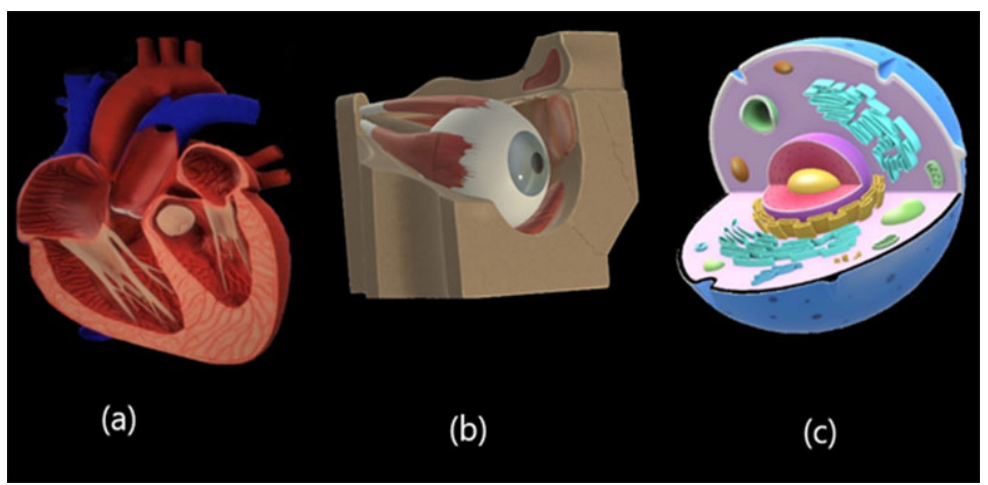

Fig. 11. The holograms created for the EduGram system

EduGram Implementation Structure: After specifying and installing the Hologram fan device and preparing the sample of the educational visual contents, the method in which this Hologram technology system component will be used in education must be specified. Figure 12 shows the chosen structure to implement EduGram system in education, the core components that must be provided in order to use Hologram technology are: (1) An installed Hologram fan device. (2) A smart device that supports Wi-Fi technology and containing an installed Hologram fan application.

According to Figure 12, that shows EduGram implementation proposed procedure in a classroom where an instructor is transferring information to a number of recipient students according to specific curriculum that contains visual information must be displayed to learners, the scenario to use Hologram technology in EduGram system is as follow: In the beginning, the instructor must have a smart device in which the Hologram fan controlling app "3D LED FAN" is installed, the smart device is then connected through Wi-Fi to the Hologram fan device located in front of learners in the classroom.

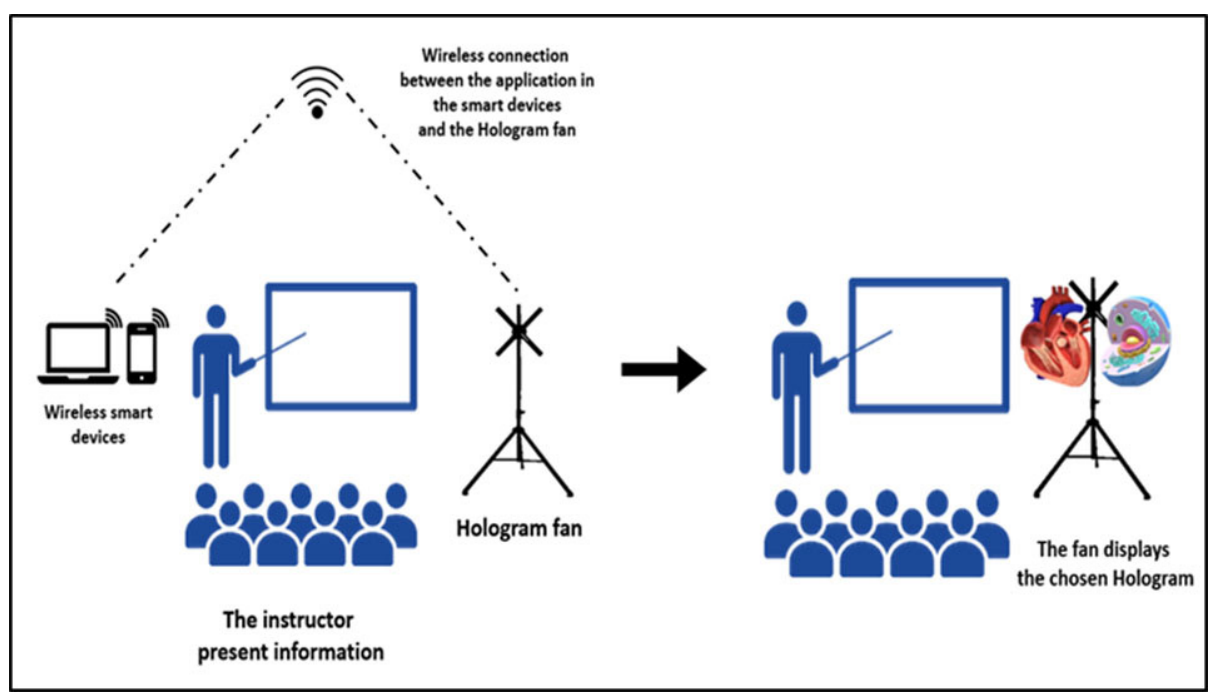

Fig. 12. EduGram implementation proposed procedure 
The 3D educational visual content must be previously stored in the memory inserted in the fan device if not, the instructor then send the content from the smart device to the fan through the application, the instructor provides information normally and continues to teach learners, and in case that there is visual information that must be displayed, he/ she displays it on the Hologram fan device and then controls its display and the rest of the visual content that it will display to students, as soon as the Hologram fan device receives the command, the Hologram of the selected visual information is displayed.

\section{$5 \quad$ Result and discussions}

After we completed EduGram procedures and all requirements defined, and after preparing the Edugram's samples, we published the 3D models that we created by placing them in a survey in addition to a number of questions to a simple sample of people for the purpose of measuring the community's awareness about hologram technology and to see the level of their acceptance of using it as an educational tool, In addition to knowing whether the hologram technique gives them a more clear educational content. So, we conducted a simple survey, which was answered by people of different ages of both females and males. The survey was distributed to the community around here in the Kingdom of Saudi Arabia, the following is the questions formula and results obtained of the survey.

At first, we wanted to determine the number of respondents who are students or anyone who is in an educational path, so we asked about the respondent current relationship to education. The results illustrate that $36 \%$ of people answered that they are students, $19 \%$ said they had children in educational levels, and $16 \%$ said they were teachers, while the rest were people interested in technology. In the next question, they were asked: "Did you hear about hologram technology?" And their answers were as follows: the blue part represents $64.2 \%$ of the respondents who answered yes, which means that they heard about it, while the red part is $35.8 \%$ who did not hear about it.

For the people who said that they heard about hologram technology, we asked them "If you answered that you heard about Hologram technology, then what is your level of knowledge of it?" in order to determine their level of knowledge about hologram technology. $38.2 \%$ of the people had no knowledge about the hologram technology but only knew its name. and the results show that more than half of the respondents have some knowledge about hologram technology, and $6 \%$ of the respondents have sufficient information about Hologram technology.

The next question was initially included a video clip explaining how the 3D heart will look like when it displayed on the Hologram fan, and next to it, the 2D image of the heart taken from the textbook. After the respondent compared the two methods, we asked a question to people to find out which method was the best for them and the question formula was: "Which method was the clearer for you?". $96.2 \%$ of people preferred the hologram model, while $3.8 \%$ preferred the $2 \mathrm{D}$ image.

The last question in the survey was to measures how much people want to apply and use Hologram technology in their lives compared to the augmented reality technology that is somewhat similar to the Hologram technology, and the question formula is: "Which of the two technologies attracts your attention more and you would like to try and use it?". 75.6\% from the respondents and who represent the majority said that they prefer the use of Hologram technology. 
The results of the survey encourage us to contribute to the development of hologram technology usage and use it as an educational tool. The results also show that there is a great and good opportunity that people accept the use of the Hologram technology, and then it can be used in their lives and in various fields such as education, which is one of the rich fields that Hologram technology can contribute to its development.

\section{Conclusion}

Education is a very important field that leads to the development of people's lives, it was necessary to use images and visual contents in the education process as humans are visual creatures, and a large percentage of the human brain dedicated itself to visual processing, they are always seeking the easiest and most fluent way of acquiring and learning information. The traditional way of educating and the used visual education content includes some problems that affect students' learning. Hologram technology and Augmented Reality are two technologies that display visual objects as 3D images in the user environment, they could be used as an educational tool to present the visual information to students in education in order to develop the information transferring process. Because the two technologies produce almost the same result and people usually confuse them, school officials may ask which technology is considered as the best option to be used in teaching students to obtain a successful educational process, so they were compared to determine the best option to be used in education.

The result of the competition showed the effectiveness of Hologram technology to be used in education, and in view of the many advantages that Hologram technology possesses, the goal of this research was to is taking advantage of this technology and develop the education by using Hologram projector as a visual displaying tool that replaces the traditional methods used, we chose the Hologram fan device to be the projector device that we use to study the development of education, because it has many capabilities, such as the ability to respond to control through software applications wirelessly and many other advantages. To implement the system, we selected samples from the science textbooks and then we designed three-dimensional models for it to be displayed on the device, then compared it to the old used images in the textbooks. We adopted on the results of the questionnaire that we distributed to a sample of people via the Internet, to determine which methods of displaying visual information in education they prefer and believe that it is the best, the results of the questionnaire were clear and decisive for the fact that Hologram technology has evolved from the process of transferring information significantly, which could contribute to developing education significantly and affects its returns positively.

\section{$7 \quad$ References}

[1] Education. (n.d.). Wikipedia. Retrieved September 27, 2021, from https://en.wikipedia.org/ wiki/Education

[2] El-Seoud, A., Halabi, O., and Geroimenko, V. (2019). Assisting individuals with autism and cognitive disorders: an augmented reality-based framework. International Journal of Online and Biomedical Engineering (iJOE), 15(4): 28-39. https://doi.org/10.3991/ijoe.v15i04.9835 
[3] Hutton, Xunzi, E. L. The complete Text. princeton university press. (n.d.). Jstor. Retrieved September 27, 2021, from http://www.jstor.org/stable/j.ctt6wq19b

[4] Balm, J. The power of pictures. How we can use images to promote and communicate science. (2014). Biomedcentral. http://blogs.biomedcentral.com/bmcblog/2014/08/11/ the-power-of-pictures-how-we-can-use-images-to-promote-and-communicate-science/

[5] Patrão, B., and Menezes, P. (2013). A virtual reality system for training operators. International Journal of Online Engineering (iJOE), 9(S8): 53-55. https://doi.org/10.3991/ijoe. v9iS8.3383

[6] Alghamdi, A. (2020). Analyzing the barriers and possibilities with p-values towards starting a new postgraduate computer and engineering programs at Najran university: A cross-sectional study. International Journal of Advanced Computer Science and Applications (IJACSA), 11(12). https://doi.org/10.14569/IJACSA.2020.0111215

[7] Holography. (n.d.). Wikipedia. Retrieved September 27, 2021, from https://en.wikipedia. org/wiki/Holography

[8] Elmahal, D. M., Ahmad, A. S., Alomaier, A. T., Abdlfatah, R. F., and Hussein, D. M. (2020). Comparative study between hologram technology and augmented reality. Journal of Information Technology Management, 12(2): 90-106.

[9] Mawardani, A., Dwandaru, W. S. B., and Nugroho, M. P. Multimedia Learning Module (MLM) with Hologram Simulation to Improve Students' Mathematical Representation Ability. In 6th International Seminar on Science Education (ISSE 2020), March 2021, Atlantis Press, pp. 522-527. https://doi.org/10.2991/assehr.k.210326.075

[10] Naveed, Q. N., Qureshi, M. R. N. M., Shaikh, A., Alsayed, A. O., Sanober, S., and Mohiuddin, K. (2019). Evaluating and ranking cloud-based e-learning critical success factors (CSFs) using combinatorial approach. IEEE Access, 7: 157145-157157. https://doi. org/10.1109/ACCESS.2019.2949044

[11] Orcos, L., Jordán, C., and Magreñán, A. (2019). 3D visualization through the hologram for the learning of area and volume concepts. Mathematics, 7(3): 247. https://doi.org/10.3390/ math 7030247

[12] What is 3d? (n.d.). Mediacollege. Retrieved September 27, 2021, from https://www. mediacollege.com/3d/intro.html

[13] Ooi, E. H., and Archarya, U. R. (2008). A comparative study between the two-dimensional and three-dimensional human eye models. Mathematical and Computer Modelling, 48: 712-720. https://doi.org/10.1016/j.mcm.2007.11.011

[14] Turk, H., and Seckin-Kapucu, M. (2021). Innovative technology applications in science education: Digital holography. Journal of Education in Science, Environment and Health, 7(2): 156-170.

[15] Elmarash, G. A., Adrah, M. M., and Eljadi, E. E. (2021). 3D hologram technology in Libyan educational institutions in future: Re-view. Journal of Pure and Applied Sciences, 20(3): 6-10. https://doi.org/10.51984/jopas.v20i3.1000

[16] Slinger, C., Cameron, C., and Stanley, M. (2005). Computer-generated holography as a generic display technology. Computer, 38(8): 46-53. https://doi.org/10.1109/MC.2005.260

[17] Hologram. (n.d.). Merriam-Webster. Retrieved September 27, 2021, from https://www. merriam-webster.com/dictionary/Hologram

[18] Dennis gabor, Father of holography. (n.d.). Bbvaopenmind. Retrieved September 27, 2021, from https://www.bbvaopenmind.com/en/science/leading-figures/dennis-gabor-fatherof-holography/

[19] Holograph vs. Hologram - what's the difference? (n.d.). Ask Difference. Retrieved September 27, 2021, from https://www.askdifference.com/holograph-vs-Hologram/ 
[20] Algarawi, F. K., Alslamah, W. A., Alhabib, A. A., Alfehaid, A. S., and Ibrahim, D. M. (2018). Applying augmented reality technology for an e-learning system. International Journal of Computer and Information Engineering, 12(3): 182-187.

[21] Fairuz, M., Jamali, S., and Wong, K. (2014). A review of augmented reality and mobile-augmented reality technology. The International Journal of Learning in Higher Education, 20: 37-54. https://doi.org/10.18848/2327-7955/CGP/v20i02/48690

[22] The difference between virtual reality (vr) and augmented reality (ar). (n.d.). Magic-Holo. Retrieved September 27, 2021, from https://magic-holo.com/en/difference-betweenvirtual-reality-vr-and-Augmented-reality-ar/

[23] Holograms add new dimension to holocaust survivor's story. (2019). N. Universal. https:// www.today.com/series/are-we-there-yet/Holograms-add-new-dimension-holocaustsurvivors-story-t20511

[24] Pimax - VRStation. (2019). VRStation. https://vrstation.id/tag/pimax

[25] Maier, P., and Klinker, G. (2013). Augmented chemical reactions: 3D interaction methods for chemistry. International Journal of Online Engineering (iJOE), 9(S8): 80-82. https://doi. org/10.3991/ijoe.v9iS8.3411

[26] S. A. (2018). How wearables, augmented reality and virtual reality will make a difference in education - rAVe [PUBS]. Www.Ravepubs.Com. https://www.ravepubs.com/ wearables-augmented-reality-virtual-reality-education/

[27] École d'éducation à l'innovation Maternelle. Hologramme sur le futur centre des musées. (n.d.). Freepik Company. Retrieved September 27, 2021, from https://fr.freepik.com/ vecteurs-premium/ecole-education-innovation-maternelle-hologramme-futur-centremusees $5234823 . \mathrm{htm}$

[28] Z.T. (2018b). 10 best real-world applications of hologram technology. Interesting Engineering. https://interestingengineering.com/10-best-real-world-applications-of-hologramtechnology

[29] 3D holographic led fan display. (n.d.). Afriten. Retrieved September 27, 2021, from https:// afriten.co.za/portfolios/3d-holospin-3d-holographic-led-fan-display/

[30] Amazon.com: Giwox upgrade holo65 3d hologram fan display, 25.6inch large size and 960p high pixel, 960 led beads and audio,holographic advertising is perfect for trade show,store display: Electronics. (n.d.). Amazon. Retrieved September 27, 2021, from https://www.amazon.com/GIWOX-Projector-Detachable-Hi-Resolution-Holographic/dp/B07HVLRX5C/ $\underline{\text { ref }=\text { olp product details? encoding }=\mathrm{UTF} 8 \& \mathrm{me}=\& \mathrm{th}=1}$

\section{Authors}

Asma Suliman is Eritrean Information Technology Graduate, Qassim University, Saudi Arabia Master's Student in Information Technology, Member of the Women in Technology (WIT) Group, Member of the winning team of the Innovation Award in Graduation Projects at Qassim University 2020 (email: asma20se@gmail.com).

Alaa Talal is Syrian Information Technology Graduate, Qassim University, Kingdom of Saudi Arabia Master's Student in Information Technology, Member of the winning team of the Innovation Award in Graduation Projects at Qassim University 2020 (email: alaa.talal.29701@gmail.com).

Doaa Elmahal is Sudanese Information Technology Graduate, Qassim University, Saudi Arabia, Master's Student in Information Technology, Alimam Alhadi college Sudan. Member of the Women in Technology (WIT) Group and Coder making group. 
Member of the winning team of the Innovation Award in Graduation Projects at Qassim University 2020 (email: doaaelmahal@gmail.com).

Reem Fahmi is an Egyptian Information Technology Graduate, Qassim University, Kingdom of Saudi Arabia. She got a diploma in Software Engineering from an information technology institution (ITI), Egypt. Master's student in information technology, Mansoura University, Egypt.Member of the winning team of the Innovation Award in Graduation Projects at Qassim University 2020 (email: reemfahmi9@gmail.com).

Dina M. Ibrahim is an assistant professor at the department of Information Technology, College of Computer, Qassim University, Buraydah, Saudi Arabia from September 2015 till now. In addition, Dina works as Lecturer at the Computers and Control Engineering Department, Faculty of Engineering, Tanta University-Egypt. She was born in the United Arab of Emirates, her B.Sc., M.Sc., and Ph.D. degrees taken from the Computers and Control Engineering Department-Faculty of Engineering, Tanta University in 2002, 2008, and 2014, respectively. Dina works as a Consultant Engineer, then a Database administrator, and finally acts as a Vice Manager on Management Information Systems (MIS) Project, Tanta University, Egypt, from 2008 until 2014. Her research interests include networking, wireless communications, machine learning, security, and the Internet of Things. Dina has published more than 42 articles in various refereed international journals and conferences. She is serving as a reviewer in Wireless Network (WINE) the Journal of Mobile Communication, Computation, and Information since 2015 Dina also acts as a Co-Chair of the International Technical Committee for the Middle East Region of the ICCMIT conference since 2020 (email: d.hussein@qu.edu.sa, dina.mahmoud@,f-eng.tanta.edu.eg).

Article submitted 2021-09-06. Resubmitted 2021-10-11. Final acceptance 2021-10-12. Final version published as submitted by the authors. 